\title{
Unified approach to hard diffraction
}

\author{
R.Peschanski \\ Service de Physique Théorique, CEA, CE-Saclay \\ F-91191 Gif-sur-Yvette Cedex, France
}

\begin{abstract}
Using a combination of S-Matrix and perturbative QCD properties in the small $x_{B j}$ regime, we propose a formulation of hard diffraction unifying the partonic (Ingelman-Schlein) Pomeron, Soft Colour Interaction and QCD dipole descriptions.
\end{abstract}

\section{Introduction}

In the present paper, we focus on three existing different theoretical approaches of hard diffraction, for which we propose a new, unifying formulation. The first one we will refer to is the "partonic Pomeron" approact . The hard photon is here supposed to probe the parton distributions of the Pomeron Regge pole considered as a hadronic particle. A second approacla is the Soft Colour Interaction one, where hard diffraction is described by a two-step process: during a relatively short "interaction time", the probe initiates a typical hard deepinelastic interaction. Then, at large times/distances, a soft colour interaction is assumed which will decide of the separation between diffractive and nondiffractive events according to a simple probabilistic rule. A third approach is based on the small $x_{B j}$ regime of perturbative QCD, where the resummation of leading $\log 1 / x_{B j}$ leads to the "QCD dipole" approach for hard diffractioli.

We show that the three aproaches may find a common formulation. Our main results are the following:

1) The "effective" parameters of the partonic Pomeron are determined from leading log perturbative QCD resummation. They are found to depend not only on $Q^{2}$ but also on the ratio $\eta=(Y-y) / y$, where $\mathrm{Y}$ (resp. y) are the total (resp. gap) rapidity interval.

2) The "effective" parameters of the hard process before modification by the Soft Colour Interaction are determined. Differences with the original model avoid the "Low's theorem" paradoll.

3) Using S-Matrix properties of triple-Regge contributions, a relation is found between discontinuities of a $3 \rightarrow 3$ amplitude and the three approaches to hard diffraction. In particular, a theoretically founded formulation of a (modified) Soft Colour Interaction approach is proposed as a specific double discontinuity the $3 \rightarrow 3$ forward amplitude. 


\section{From QCD dipoles to partonic Pomeron}

Our starting point is the formula for the structure function (inelastic component) for longitudinal and transverse photon:

$$
\begin{gathered}
F_{T, L}^{D i f f}\left(Q^{2}, Y, y\right) \sim \int_{c-i \infty}^{c+i \infty} \frac{d \gamma_{1}}{2 i \pi} \frac{d \gamma_{2}}{2 i \pi} \frac{d \gamma}{2 i \pi} \delta\left(1-\gamma_{1}-\gamma_{2}-\gamma\right) \\
\times\left(\frac{Q}{Q_{0}}\right)^{2 \gamma} \exp \left\{y\left[\Delta\left(\gamma_{1}\right)+\Delta\left(\gamma_{2}\right)\right]+(Y-y)[\Delta(\gamma)]\right\}
\end{gathered}
$$

where

$$
\Delta(\gamma) \equiv \frac{\alpha_{s} N_{c}}{\pi}\{2 \psi(1)-2 \psi(\gamma)-2 \psi(1-\gamma)\} \sim \Delta+\frac{\Delta^{\prime \prime}}{2}\left(\frac{1}{2}-\gamma\right)^{2}
$$

is the BFKL evolution kernel (used in a gaussian approximation), $Q_{0}$ a nonperturbative scale associated with the proton. After a triple saddle-point approximation (using the gaussian approximation) justified by the large rapidity gap characteristic of diffraction, one may write $F_{T, L}^{D i f f} \sim e^{2 y \Delta} \sigma_{\gamma^{*}-P}^{t o t}$, with

$$
\sigma_{\gamma^{*}-P}^{t o t} \sim \sqrt{\frac{2}{1+2 \eta}} \exp \left\{(Y-y) \epsilon_{s}\right\}\left(\frac{Q}{Q_{0}}\right)^{2 \gamma_{\mathbf{s}}} \exp \left(-\frac{2 \eta}{1+2 \eta} \frac{2 \log ^{2}\left(\frac{Q}{Q_{0}}\right)}{\Delta^{\prime \prime}(Y-y)}\right)
$$

Here, the gaussian saddle-points determine both the new "effective dimension" and "effective intercept":

$$
\gamma_{\mathbf{s}}=\frac{\eta}{1+2 \eta} ; \epsilon_{s}=\Delta+\frac{\Delta^{\prime \prime}}{8(1+2 \eta)}
$$

of a "BFKL-like" expression, depending on the variable $\eta=\frac{Y-y}{y}$.

$\sigma_{\gamma^{*}-P}^{t o t}$ thus defines an effective "partonic Pomeron" derived from the QCD dipole formalism.

\section{From dipoles to Soft Colour Interaction}

In Soft Color Interactions models, at least in their formally simpler formulations, one expects the following relation between the total structure function and the overall contribution of hard diffraction at fixed value of $x_{B j}$ :

$$
F_{T, L}^{D i f f / t o t} \equiv \int_{x_{B j}}^{x_{g a p}} d x_{P} F_{T, L}^{D i f f}=\frac{\mathbf{1}}{\mathbf{N}_{\mathbf{c}}^{\mathbf{2}}} F_{T, L}^{\text {hard } / \text { tot }}
$$


where $\log \frac{1}{x_{\text {gap }}}$ is the minimal value retained for the rapidity gap. A priori, $F_{T, L}^{\text {hard/tot }}$ is supposed not to be modified by the soft color interaction. However a contradiction with "Low's theorem" has been quoted. Indeed, a fully soft color interaction can be proven to give no effect on physical obervables. finds

In fact, when inserting the QCD dipole expression (1) in relation (5), one

$$
F_{L, T}^{\text {hard } / \text { tot }} \sim\left(\frac{Q}{Q_{0}}\right)^{2 \gamma_{\text {hard }}} \frac{\exp \left(Y \Delta\left(\gamma_{\text {hard }}\right)\right)}{\sqrt{2 \pi \Delta^{\prime \prime} Y}}
$$

which is very similar with the BFKL expression, except that

$$
\gamma_{B F K L} \sim \frac{1}{2}\left(1-4 \frac{\log \left(\frac{Q}{Q_{0}}\right)}{\Delta^{\prime \prime} Y}\right) \neq \gamma_{h a r d}=c s t \sim 0.175 .
$$

Thus, in some sense, the Soft Color relations are compatible with QCD dipole calculations, but the underlying hard interaction is somewhat different than the assumed mechanism in SCI models. Hence the soft color rearrangement is not simply a "neutralization" of color at long distances which would be forbidden by virtue of "Low's theorem". Apart this modification, formula (5) fixes the relative normalization between diffractive and total structure functions. .

\section{S-Matrix interpretation}

Following old results of S-Matrix theory in the Regge domain 6 , and as sketched in the figure, one may consider three types of discontinuities of a $3 \rightarrow 3$ amplitude representing hard diffraction. A single discontinuity over the diffractive $m a s s^{2}$ variable, a double discontinuity taking into account also the analytic discontinuity in the subenergy of one of the incident Pomeron exchanges and a triple discontinuity with the discontinuity including the two Pomeron exchanges.

As qualitatively shown in the figure, one can associate the three discontinuity formulae with, respectively, the partonic Pomeron, Soft Color Interaction and QCD dipole approaches. This is schematically depicted in the "timedependent" representation of the figure, where the soft incident Pomerons are represented as living during a "long" time, while the partonic (or QCD dipole) process are initiated by hard interactions. In fact, one can prove the identity of these discontinuities, and thus legitimate the connection realized in the previously mentionned calculations. 

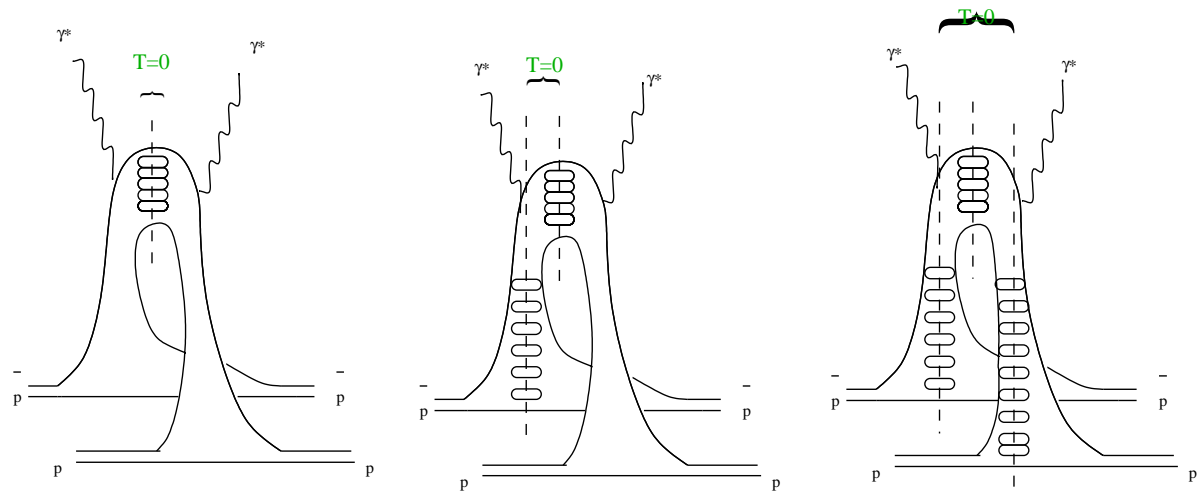

Figure 1: "Time-dependent" picture of the $A(3 \rightarrow 3)$ discontinuities. Upper graph: Description of $\operatorname{Disc}_{1} A(3 \rightarrow 3)$ (partonic Pomeron approach); Middle graph: Description of $D i s c_{2} A(3 \rightarrow 3)$ (candidate for the Soft Colour Interaction approach); Lower graph: Description of $\operatorname{Disc}_{3} A(3 \rightarrow 3)$ (QCD dipole approach).

\section{Acknowledgments}

This work was done in collaboration with H. Navelet.

\section{References}

1. G. Ingelman, P. Schlein, Phys. Lett. B152 (1985) 256.

2. W. Buchmuller, A. Hebecker, Phys. Lett. B355 (1995) 573; A. Edin, G. Ingelman, J. Rathsman, Z. Phys. C75 (1997) 57.

3. A. Bialas, R. Peschanski, Phys. Lett. B378 (1996) 302; B387 (1996) 405.

4. We thank Y. Dokshitzer for pointing out to me the problem that a truely soft color interaction should not modify the physical observables (cf. "Low's theorem").

5. H. Navelet, R. Peschanski, hep-ph/0105030.

6. A. Mueller, Phys. Rev. D2 (1970) 2963, D4 (1971) 150; R.C. Brower, C.E. DeTar, J.H. Weis, Phys. Rept. 14 (1974) 257. 